\title{
Effects of cocaethylene on dopamine and serotonin synthesis in Long-Evans and Sprague-Dawley brains
}

\author{
Michael H. Baumann ${ }^{\text {a }}$, Judith M. Horowitz ${ }^{\text {b }}$, Mark B. Kristal ${ }^{\text {b }}$, German Torres ${ }^{\text {b, * }}$ \\ ${ }^{a}$ Clinical Psychopharmacology Section, Intramural Research Program, National Institute on Drug Abuse, National Institutes of Health, Baltimore, MD \\ 21224, USA \\ ${ }^{\mathrm{b}}$ Behavioral Neuroscience Program, Department of Psychology, State University of New York at Buffalo, Buffalo, NY 14260, USA
}

Accepted 7 July 1998

\begin{abstract}
We examined the behavioral and neurochemical effects of cocaethylene treatment in Long-Evans (LE) and Sprague-Dawley (SD) rats. Cocaethylene-induced behaviors were significantly less in LE rats. Cocaethylene caused an inhibition of dopamine synthesis in the caudate nucleus and nucleus accumbens that was equivalent in both rat lines. Serotonin synthesis was also suppressed by cocaethylene treatment, however this phenomenon was less pronounced when compared with the effects on dopamine synthesis. (C) 1998 Published by Elsevier Science B.V. All rights reserved.
\end{abstract}

Keywords: Behavior; Caudate nucleus; Cingulate cortex; Cocaine; Genotype; Nucleus accumbens

Concurrent intake of cocaine and ethanol results in the formation of a pharmacologically active cocaine metabolite, cocaethylene [14]. Studies show that cocaethylene is synthesized in vivo by the actions of liver carboxylesterase enzymes in both rats and humans [2,4]. Cocaethylene, like cocaine, binds to dopamine (DA) transporter sites in the brain parenchyma thereby inhibiting the reuptake of DA molecules into pre-synaptic neurons [6,9]. In contrast to cocaine, cocaethylene displays a relatively low affinity for serotonin (5-HT) transporters and is therefore more DAspecific in its actions than its parent compound. Administration of cocaethylene to rats elicits behaviors such as increased locomotor activity, stimulation of operant responses and context-dependent sensitization $[10,13,15]$. Similarly, in humans cocaethylene produces a myriad of subjective effects, including euphoria, that closely resemble those elicited by cocaine $[11,12]$.

We have found that Long-Evans (LE) rats show less behavioral sensitivity to cocaethylene than do SpragueDawley (SD) rats [8]. This fact is particularly intriguing since differences in behavioral sensitivity appear to be specific to cocaethylene but not cocaine treatment [8]. The

\footnotetext{
* Corresponding author. Fax: + 1-716-645-3801; E-mail: gtorres@acsu.buffalo.edu
}

factors responsible for the strain-dependent variation in responsiveness to cocaethylene are not known but could involve: (i) strain differences in cocaethylene bioavailability or, (ii) strain differences in DA and 5-HT transmission in discrete brain regions mediating behavioral activity. The purpose of the present study was therefore to test the latter possibility by examining the effects of acute cocaethylene treatment on DA and 5-HT synthesis in various brain regions of $\mathrm{LE}$ and $\mathrm{SD}$ rats. We used the technique of measuring L-3,4-dihydroxyphenylalanine (L-DOPA) and L-5-hydroxytryptophan (5-HTP) accumulation after inhibition of aromatic L-amino acid decarboxylase with 3-hydroxybenzylhydrazine (NSD-1015), as originally described by Carlsson et al. [3]. With this method, L-DOPA and 5-HTP levels measured in postmortem brain tissue reflect the in vivo activities of tyrosine hydroxylase (for DA synthesis) and tryptophan hydroxylase (for 5-HT synthesis), respectively.

Adult male LE (born and raised in our laboratories) and SD rats (purchased from Harlan; 220-260 g) were maintained on a 14:10 h light:dark (L:D) cycle with free access to food and water. To minimize the possibility of nonspecific stress on monoamine synthesis, rats were handled for three days prior to any experimental manipulation. Drug injections were administered between 1000 and $1200 \mathrm{~h} \mathrm{(4}$ $\mathrm{h}$ after the onset of the light phase). Testing procedures 
were carried out in accordance with the NIH Guide for the Care and Use of Laboratory Animals, and with approval from the State University of New York at Buffalo IACUC. Cocaethylene fumarate was dissolved in sterile saline and injected intraperitoneally (i.p.), at a dose of $20 \mathrm{mg} / \mathrm{kg}$ (calculated as the free base). Control rats received i.p. saline-vehicle injections $(1 \mathrm{ml} / \mathrm{kg})$. The dose of cocaethylene was selected because it elicits a robust increase in behavioral activity in SD rats [16,17]. Behaviors were rated just before the first injection (cocaethylene or saline) and every $5 \mathrm{~min}$ for $30 \mathrm{~min}$ thereafter using a behavioral rating scale described previously [8]. Thirty minutes after cocaethylene or saline injections, all rats received NSD1015 (RBI, Natick, MA) at a dose of $100 \mathrm{mg} / \mathrm{kg}$, i.p. The dose of NSD-1015 was selected based on previous studies examining the effects of cocaine on DA and 5-HT synthesis in rat brain $[1,5]$. Thirty min after NSD-1015 (i.e., 60 min after cocaethylene or saline treatment), rats were killed by decapitation. Brains were immediately removed, frozen on dry ice and stored at $-80^{\circ} \mathrm{C}$ until the quantification of L-DOPA and 5-HTP by high-performance liquid chromatography with electrochemical detection. For specific details on this neurochemical procedure see Baumman et al. [1]. Behavioral data were analyzed using a Two-way ANOVA with repeated measures, whereas neurochemical data were evaluated by Two-way ANOVA. When significant $F$ values were obtained, Newman-Keuls post-hoc tests were performed to determine differences between group means. Concentrations of L-DOPA and 5-HTP from each brain region were determined and expressed as mean \pm S.E.M. (pg/mg protein). Subsequently, these values were expressed as percent control values; this was accomplished by dividing each raw value by the control group mean, multiplied by 100 .

Cocaethylene elicited a significant increase in behavioral activity $(P \leq 0.05)$ which included intense bouts of sniffing, rearing and locomotion in SD, but not in LE rats (Fig. 1). Strain-dependent differences in activity were apparent within $5 \mathrm{~min}$ of cocaethylene administration and were steadily maintained throughout the testing session. In contrast, rats of both strains injected with $1 \mathrm{ml} / \mathrm{kg}$ saline exhibited little behavioral activity (data not shown). For example, behavioral scores obtained at $15 \mathrm{~min}$ from saline-treated LE and SD rats corresponded mainly to still or in-place activity (i.e., scores of 1-2). It should be noted, however, that LE rats injected with cocaethylene invariably displayed greater behavioral activity than did cohorts injected with saline. This suggests that although LE rats are relatively insensitive to the stimulant effects of cocaethylene, the ethyl metabolite is still capable of eliciting modest bouts of behavioral activity.

Rates of L-DOPA and 5-HTP accumulation in various brain regions from saline-treated $\mathrm{LE}$ and $\mathrm{SD}$ rats are depicted in Table 1. Basal levels of L-DOPA were relatively low in the cingulate cortex, whereas levels in the caudate nucleus and nucleus accumbens were much higher.

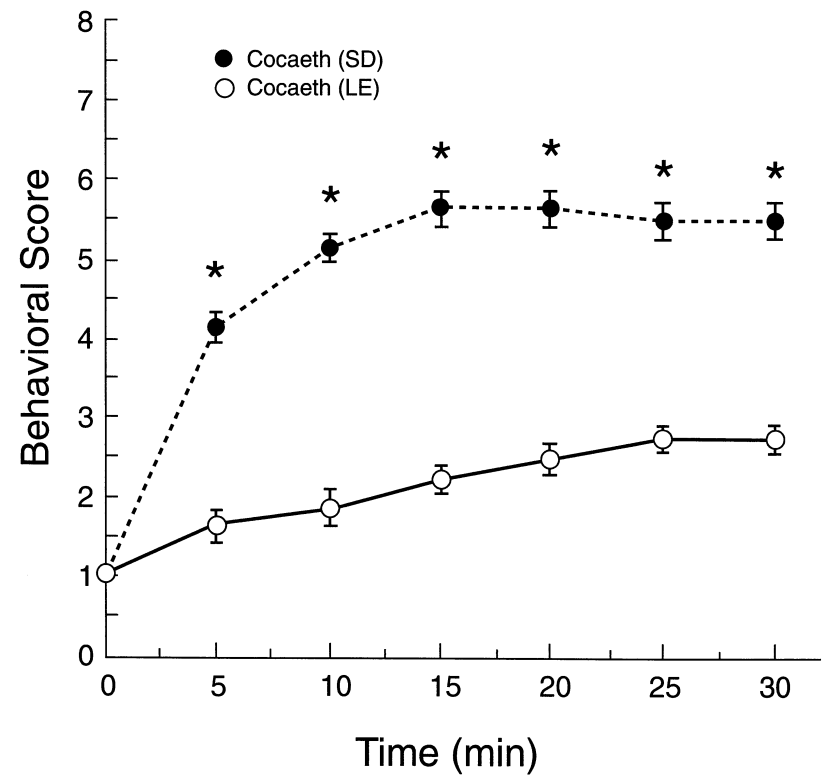

Fig. 1. Behavioral effects of cocaethylene (cocaeth) administered at 20 $\mathrm{mg} / \mathrm{kg}$ (i.p.) to LE and SD rats. Data are expressed as mean \pm S.E.M. for $n=6-7$ rats /group. SD rats (broken lines) showed a significantly greater increase in behavioral activity when compared with LE rats (solid lines). Two-way repeated measures ANOVA on one factor showed a significant main effect of drug treatment $\left(F_{3,138}=374 ; P \leq 0.0001\right)$ and a significant main effect of time $\left(F_{6,138}=60.0 ; P \leq 0.0001\right)$ as well as a significant drug treatment $\times$ time interaction $\left(F_{18,138}=24.4 ; P \leq 0.0001\right)$. $* P$ $\leq 0.05$ with respect to corresponding LE rats. Behavioral scores of 5 and 6 correspond to continuous sniffing with various degrees of locomotion and rearing.

This neurochemical profile was consistent across strains. Therefore, no significant differences in basal L-DOPA accumulation were detected in any brain region between LE and SD rats. Basal levels of 5-HTP accumulation were relatively low in the cingulate cortex and caudate nucleus, whereas transmitter levels in the nucleus accumbens were slightly higher. Again, this profile was similar in both rat phenotypes with levels of 5-HTP not significantly different between control rats of the LE and SD strain.

The effects of cocaethylene on L-DOPA accumulation are shown in Fig. 2. Administration of cocaethylene signif-

Table 1

L-DOPA and 5-HTP levels in brain regions from LE and SD control rats ${ }^{\mathrm{a}}$

\begin{tabular}{llllll}
\hline & \multicolumn{4}{l}{ LE } & \multicolumn{3}{l}{ SD } \\
\cline { 2 - 3 } \cline { 5 - 6 } & L-DOPA & $5-H T P$ & & L-DOPA & 5-HTP \\
\hline $\begin{array}{l}\text { Cingulate } \\
\text { cortex }\end{array}$ & $0.585 \pm 0.087$ & $0.518 \pm 0.043$ & & $0.528 \pm 0.021$ & $0.487 \pm 0.022$ \\
$\begin{array}{l}\text { Caudate } \\
\text { nucleus }\end{array}$ & $7.580 \pm 0.590$ & $0.590 \pm 0.054$ & $6.739 \pm 0.478$ & $0.432 \pm 0.034$ \\
$\begin{array}{l}\text { Nucleus } \\
\text { accumbens }\end{array}$ & $6.742 \pm 0.776$ & $1.097 \pm 0.134$ & $6.479 \pm 0.179$ & $1.124 \pm 0.060$ \\
\hline
\end{tabular}

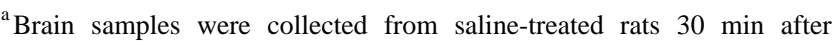
NSD-1015 treatment. Values are means \pm S.E.M. for $n=6$ rats/group. There were no significant strain-dependent differences in 5-HTP or L-DOPA levels in any brain region examined. 

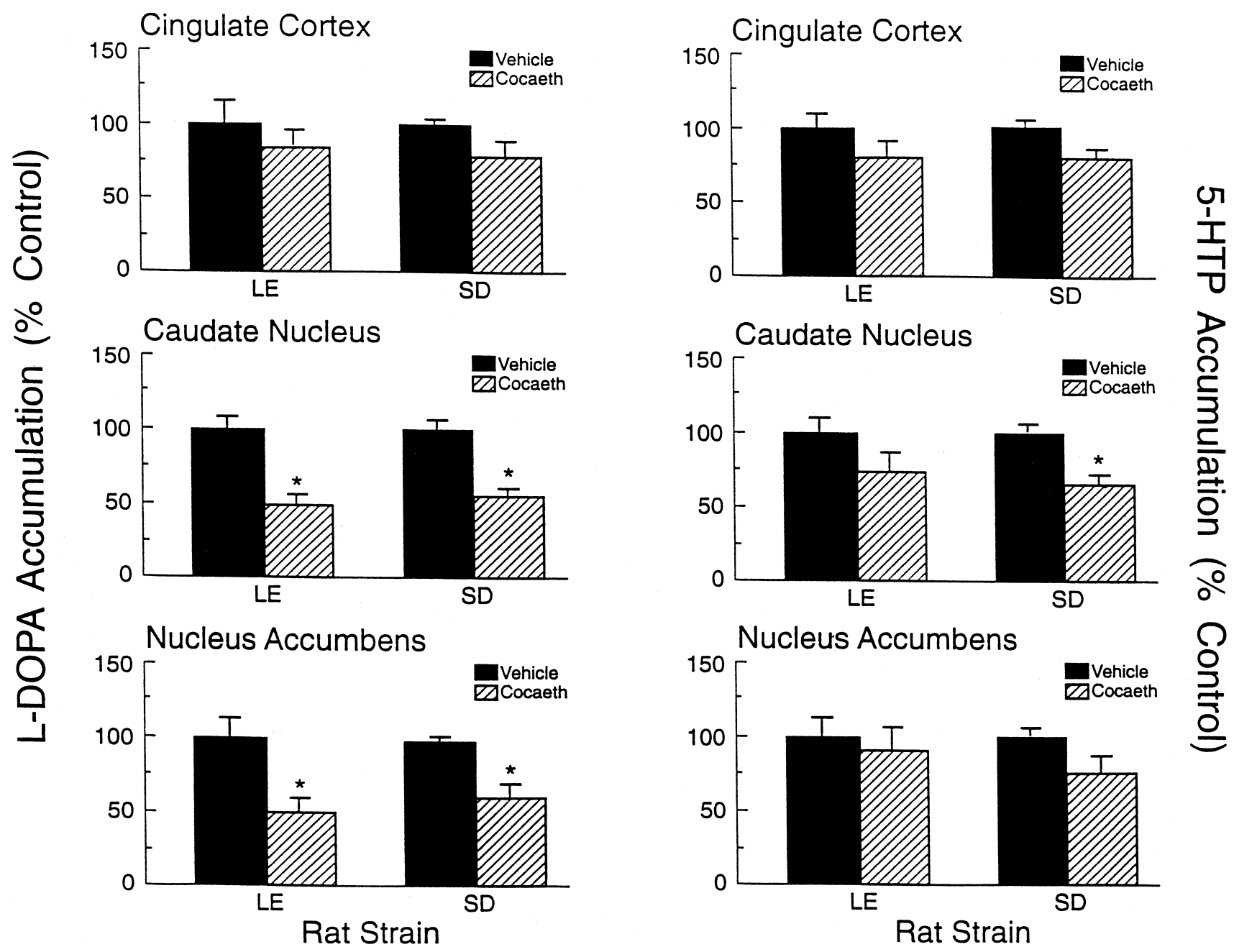

Fig. 2. Effects of cocaethylene (cocaeth) on L-DOPA and 5-HTP accumulation in microdissected brain regions of LE and SD rats. L-DOPA and 5-HTP levels are expressed as mean \pm S.E.M. for $n=6-7$ rats/group expressed as percent control of L-DOPA and 5-HTP levels determined in saline-vehicle injected rats (see Table 1). Cocaethylene produced a significant decrease in L-DOPA levels in the caudate nucleus and nucleus accumbens, but not in the cingulate cortex, of both strains. There were no strain-dependent differences in cocaethylene-induced suppression of L-DOPA accumulation. Cocaethylene produced a significant decrease in 5-HTP levels only in the caudate nucleus of SD rats. However, there were no strain-dependent differences in cocaethylene-induced effects on 5-HTP accumulation in any brain region examined. $* P \leq 0.05$ when compared with saline-vehicle injected rats of the same strain.

icantly reduced L-DOPA levels $(P \leq 0.05)$ in the caudate nucleus and nucleus accumbens of both strains. Although cocaethylene tended to decrease L-DOPA in the cingulate cortex, this effect was not statistically significant in either strain. Cocaethylene-induced reduction of L-DOPA was of equal magnitude in LE and SD rats. Indeed a Two-way ANOVA comparing L-DOPA levels between LE and SD strains indicated no significant strain-dependent differences in the cingulate cortex $\left(F_{1,22}=0.012 ; P \geq 0.05\right)$, caudate nucleus $\left(F_{1,22}=1.03 ; P \geq 0.05\right)$ or nucleus accumbens $\left(F_{1,22}=0.456 ; P \geq 0.05\right)$. In addition, ANOVA failed to show any significant effect of strain $\times$ drug interaction between the aforementioned rat lines.

As illustrated in Fig. 2, administration of cocaethylene produced modest effects on 5-HTP accumulation in LE and SD brains. Although there was a tendency for cocaeth- ylene to decrease 5-HTP levels in all brain regions across strains, this effect achieved statistical significance only in the caudate nucleus of SD brains. It is noteworthy that Two-way ANOVA revealed no strain-dependent differences in sensitivity to the effects of cocaethylene on 5-HTP in the cingulate cortex $\left(F_{1,22}=0.44 ; P \geq 0.05\right)$, caudate nucleus $\left(F_{1,22}=0.18 ; P \geq 0.05\right)$ or nucleus accumbens $\left(F_{1,22}=0.4 ; P \geq 0.05\right)$. Furthermore, ANOVA revealed no significant strain $\times$ drug interaction between the aforementioned rat lines.

We have confirmed that LE rats show less behavioral sensitivity to cocaethylene than do SD rats [7,8]. Basal rates of DA and 5-HT synthesis in various brain regions did not differ between LE and SD rats. Cocaethylene caused significant inhibition of DA synthesis in the caudate nucleus and nucleus accumbens in both strains. 
Whereas cocaethylene also tended to decrease 5-HT synthesis in LE and SD brains, this effect was relatively weak. Collectively, these data suggest that differences in DA and 5-HT transmission may not account for the differential effects of cocaethylene on behavior in LE and SD rats. We cannot dismiss the possibility, however, that fundamental differences in monoamine function (e.g., transmitter release mechanisms or post-synaptic receptor sensitivity) do exist in these rat strains but were not detected by the methods used in the present experiments. Further studies are warranted to determine the neurobiological substrate(s) mediating the behavioral insensitivity to cocaethylene observed in LE rats.

\section{Acknowledgements}

This work was supported in part by a Research Developmental Fund from the Dean of the Faculty of Social Sciences at the State University of New York at Buffalo to German Torres. The authors thank the National Institute on Drug Abuse for providing cocaethylene fumarate.

\section{References}

[1] M.H. Baumann, T.J. Raley, J.S. Partilla, R.B. Rothman, Biosynthesis of dopamine and serotonin in the rat brain after repeated cocaine injections: a microdissection mapping study, Synapse 14 (1993) $40-50$.

[2] C.S. Boyer, D.R. Petersen, Enzymatic basis for the transesterification of cocaine in the presence of ethanol: evidence for the participation of microsomal carboxylesterases, J. Pharmacol. Exp. Ther. 260 (1992) 939-946.

[3] A. Carlsson, W. Kehr, M. Lindqvist, T. Magnusson, C.V. Atack, Regulation of monoamine metabolism in the central nervous system, Pharmacol. Rev. 24 (1972) 371-384.

[4] R.A. Dean, C.D. Christian, R.H.B. Sample, W.F. Bosron, Human 225-232. liver cocaine esterases: ethanol-mediated formation of ethylcocaine, FASEB J. 5 (1991) 2735-2739.

[5] M.P. Galloway, Regulation of dopamine and serotonin synthesis by acute cocaine administration, Synapse 6 (1990) 63-72.

[6] W.L. Hearn, D.D. Flynn, G.W. Hime, S. Rose, J.C. Cofino, E. Mantero-Atienza, C.V. Wetli, D.C. Mash, Cocaethylene: a unique cocaine metabolite displays high affinity for the dopamine transporter, J. Neurochem. 56 (1991) 698-701.

[7] J.M. Horowitz, J.M. DiPirro, M.B. Kristal, G. Torres, Dopaminergic and glutamatergic mechanisms mediate the induction of FOS-like protein by cocaethylene, Brain Res. Bull. 42 (1997) 393-398.

[8] J.M. Horowitz, M.B. Kristal, G. Torres, Differential behavioral responses to cocaethylene of Long-Evans and Sprague-Dawley rats: role of serotonin, Synapse 26 (1997) 11-21.

[9] P. Jatlow, J.D. Elsworth, C.W. Bradberry, G. Winger, J.R. Taylor, R. Russell, R.H. Roth, Cocaethylene: a neuropharmacologically active metabolite associated with concurrent cocaine-ethanol ingestion, Life Sci. 48 (1991) 1787-1794.

[10] J.L. Katz, P. Terry, J.M. Witkin, Comparative behavioral pharmacology and toxicology of cocaine and its ethanol-derived metabolite, cocaine ethyl-ester (cocaethylene), Life Sci. 50 (1992) 13511361.

[11] E.F. McCance, L.H. Price, T.R. Kosten, P.I. Jatlow, Cocaethylene: pharmacology, physiology and behavioral effects in humans, J. Pharmacol. Exp. Ther. 274 (1995) 215-223.

[12] M. Perez-Reyes, A.R. Jeffcoat, Ethanol/cocaine interaction: cocaine and cocaethylene plasma concentrations and their relationship to subjective and cardiovascular effects, Life Sci. 51 (1992) 553-563.

[13] E.P.M. Prinssen, M.S. Kleven, W. Koek, Repeated administration of cocaethylene induces context-dependent sensitization to its locomotor effects, Psychopharmacology 124 (1996) 300-305.

[14] F.K. Rafla, R.L. Epstein, Identification of cocaine and its metabolites in human urine in the presence of ethyl alcohol, J. Anal. Toxicol. 3 (1979) 59-63.

[15] M.D. Schechter, Cocaethylene produces discriminative stimulus properties in the rat: effect of cocaine and ethanol coadministration, Pharmacol. Biochem. Behav. 51 (1995) 285-289.

[16] G. Torres, J.M. Horowitz, Individual and combined effects of ethanol and cocaine on intracellular signals and gene expression, Prog. Neuro-Psychopharmacol. Biol. Psychiatry 20 (1996) 561-596.

[17] G. Torres, J.M. Horowitz, S. Lee, C. Rivier, Cocaethylene stimulates the secretion of ACTH and corticosterone and the transcriptional activation of hypothalamic NGFI-B, Mol. Brain Res. 43 (1996) 